\title{
Prevalência de alterações da mucosa bucal em adolescentes brasileiros institucionalizados em dois centros de reeducação
}

\author{
Prevalence of oral mucosal alterations in Brazilian adolescents \\ held in two juvenile re-education centers
}

Ramon Aluâne H ipólito ${ }^{1}$

Carlos Roberto M artins ${ }^{2}$

\footnotetext{
${ }^{1}$ Instituto deCiências Biológicas eda Saúdeda Pontifícia Universidade Católica de M inas Gerais. Avenida Dom JoséGaspar 500, Coração Eucarístico. 30535-610 Belo Horizonte MG.

ramonaluane@gmail.com

${ }^{2}$ Faculdade deO dontologia, Pontifícia Universidade Católica de M inas Gerais.
}

Abstract Thepurpose of the present study was to verify the prevalence of oral mucosal alterations in Brazilian adolescents institutionalized. A total of 231 adolescents, all male were examined. The criteria used for clinical diagnostic of the lesionsweretheformer proposed by SB 2000 (Brazil). Thetotal oral mucosal lesions prevalencewas $27.70 \%$ (64 lesions). The total prevalence of buccal mucosal lesions were $27.70 \%$ (64 lesions) in $24,24 \%$ of the adolescents. The most frequent one was plaque. It was found 293 mucosal alterations. $78.35 \%$ of the adolescents examined presented at least one alteration. The melanin pigmentation was the most common alteration. The data collected had been analyzed statistically through Kruskall Wallis non- parametric test and associations through Qui-Square test, considering significant level of $5 \%$. Statistical significant difference was identified concerning between basic le sions and/or mucosal alterations and race $(p=0,002)$ and skin colours and average number of the mucosal alteration $(p=0,000)$ and the present of the melanin pigmentation and leukoedema and race $(p=0,000$ and $p=0,002)$. There wasn't any statistically significant difference between average number of the mucosal lesions and race $(p=0,618)$.

Key words Adolescents, Oral mucosal lesions
Resumo 0 propósito do presente estudo foi verificar a prevalência de lesões fundamentais e alterações da normalidadena mucosa bucal deadolescentes brasileiros institucionalizados. Foram examinados 231 adolescentes, todos do gênero masculino. Os critérios clínicos de diagnóstico das lesões foram os propostos pelo SB (Brasil) 2000-Prevalência de fatores de riscos. A prevalência total de lesões da mucosa bucal foi de $27,70 \%$ (64 lesões), com $24,24 \%$ dos adolescentes as apresentando. A mais frequente foi a placa. Encontraram-se 293 alterações de normalidade, com $78,35 \%$ dos examinados apresentando pelo menos uma delas. A pigmentação melânica fisiológica foi a mais encontrada. O s dados col etados foram analisados estatisticamente através do teste não paramétrico de Kruskall-Walliseas associações pelo testeQ ui-quadrado, considerando-se nível de significância de $5 \%$. Diferença estatisticamente significante foi identificada quando se avaliou a presença delesão fundamental e/ou alteração da normalidade versus cor da pele $(p=0,002)$, entre a cor da pele e 0 número médio de alterações de normalidade $(p=0,000)$, entrea presença de pigmentação melânica fisiológica e leucoedema versus cor da pele do examinado com $p=0,000$ e $p=0,002$. Sobre o número médio delesões da mucosa versus cor da pele, não houve diferença estatisticamente significante, com $p=0,618$.

Palavras-chave Adolescente, Lesões de mucosa bucal 
Introdução

Independentemente da especialidade a quese dedica, o exame sistemático da boca é de inteira responsabilidade do cirurgião-dentista. A odontologia, como ciência e profissão de saúde, não se restringindo como antes ao cuidado dos dentes e de suas estruturas de suporte, enquadra-se atualmente na área de prevenção e diagnóstico de doenças da mucosa bucal.

Esse progresso evidente da prática odontológica que, segundo o conceito de saúde proposto pela Organização M undial daSaúde(OM S), contribui para que se atinja perfeito bem-estar psicossomático e social, decorre da preocupação maior, por parte do cirurgião-dentista, com a saúde bucal de seus pacientes.

Processos patológicos diversos, como doençasinfecciosas, inflamatórias, imunológicas, alérgicas, sistêmicas e ainda lesões traumáticas, acometem frequentemente a boca.

Estudos epidemiológicos se constituem em instrumento coletivo valioso na descrição das condições de saúde da população, na investigação de seus determinantes enas ações destinadas a alterá-las ${ }^{1}$. Além disso, são de grande valia na estomatologia clínica, pois auxiliam os profissionais na elaboração dehipóteses diagnósticas, fornecendo-Ihes dados sobre a prevalência de alterações e doenças, permitindo-Ihes então estimar a possibilidade de encontrá-las na sua prática clínica.

Os estudos de prevalência realizados em nível mundial e no Brasil, particularmente ligados a problemas de saúde pública, incidem em sua maioria sobre crianças e se referem quase sempre à cárie dental, a periodontopatias, à oclusão e aos distúrbios de desenvolvimento dentais.

$\mathrm{Na}$ literatura, observam-se estudos epidemiológicos transversais geralmente provenientes de levantamentos clínicos em faculdades de odontologia, hospitais e serviços de patologia bucal. A metodologia normalmente envolve a prevalência de algumas alterações encontradas em determinado momento (estudo transversal) e ainda contempla populações específicas, portanto, esses trabalhos não refletem como um todo os aspectos epidemiológicos de todas as alterações bucaiis ${ }^{2-3}$. São encontrados relatos sobre a prevalência de alterações da mucosa bucal em adolescentes. No entanto, em razão da falta de padronização com relação à metodologia e de critérios clínicos de diagnósticos diversos, não é possível se determinar com exatidão qual é de fato a frequência das alterações ${ }^{2}$.
Em razão de não existirem outros trabalhos na literatura sobre a prevalência de alterações da mucosa bucal em adolescentes, com a particularidade de viverem confinados e diante da necessidade de se planejar atendimento, individual ou coletivo, dessa população específica, o presente estudo teve como objetivo a determinação da prevalência de alterações da mucosa bucal em menores infratores internados em dois diferentes centros de reeducação da cidade de Belo $\mathrm{Ho}$ rizonte, M inas Gerais, Brasil.

\section{Metodologia}

Neste trabalho, a amostragem se fez por conveniência e constou de uma população de estudo composta por 231 adolescentes. 0 projeto de pesquisa foi aprovado pelo Comitê de Ética em Pesquisa (Coep) da Pontifícia Universidade Católica de M inas Gerais.

Para testar o formulário de registro proposto para o estudo principal eainda o método escolhido para a coleta de dados, realizou-se um estudopiloto, que abrangeu cerca de $10,0 \%$ (22/231) da amostra estimada. Os dados obtidos neste estudo foram aproveitados no estudo principal.

0 método utilizado nesta pesquisa foi o exame clínico de uma população de adolescentes. Para a verificação da presença ou não de lesões fundamentais foi realizado o teste Kappa, com os exames realizados em duplicata, mostrando concordância intraexaminador de $100 \%$.

Para atender aos objetivos propostos, utilizamos como instrumento de pesquisa um questionário, dividido em quatro partes: (1) dados de caracterização dos participantes do estudo; (2) exame objetivo das lesões fundamentais; (3) conduta; e (4) verificação das alterações da normalidade. A realização da coleta dos dados foi feita através de inspeção e palpação, utilizando luz natural, afastadores de madeira e equipamentos de proteção individual.

Os critérios clínicos de diagnóstico enomenclatura das lesões fundamentais adotados foram os propostos pelo Saúde Bucal (SB) Brasil (2000) - Ação Complementar - Prevalência de fatores derisco - Lesões cancerizáveis e câncer da boca M inas Gerais ${ }^{4}$. As lesões fundamentais foram assim designadas: mácula, placa, nódulo, pápula, vesícula, bolha, erosão, úlcera, fissura, pseudomembrana e hiperplásica.

Com relação às lesões fundamentais, trabaIhamos com os aspectos clínicos caracterizados por N eville et al. ${ }^{5}$ - mácula: área focal com altera- 
ção na coloração, sem elevação ou depressão em relação aos tecidos circunjacentes; placa: Iesão ligeiramenteel evadae com superfície plana; nódulos: lesões sólidas e elevadas com cinco ou mais milímetros de diâmetro; pápulas: lesões sólidas e elevadas, com menos de $5 \mathrm{~mm}$ de diâmetro; vesículas: bolhas superficiais com menos de $5 \mathrm{~mm}$ de diâmetro; bolhas: vesículas grandes com mais de $5 \mathrm{~mm}$ de diâmetro; erosão: lesão superficial que tem como característica a perda parcial da superfície epitelial; úlceras: caracterizadas pela perda da superfície epitelial com exposição do tecido conjuntivo subjacente; fissura: ulceração estreita semelhante a um sulco; pseudomembrana: úlceras recobertas por uma membrana removível; e lesões hiperplásicas: com elevações de coloração semelhanteà da mucosa circunjacente.

Neste estudo, foram também verificadas as seguintes alterações (variações) da normalidade: anquiloglossia, eritema areata migratório, fossetas da comissura labial, grânulos de Fordyce, leucoedema, língua fissurada, pigmentação melânica fisiológica, torus palatino, torus mandibular eúvula bífida. A pigmentação melânica fisiológica (mácula) e os grânulos de Fordyce (pápula) foram considerados alterações da normalidade e não lesões fundamentais.

Os exames obedeceram a uma sequência proposta por Kleinman et al. ${ }^{3}$. As estruturas anatômicas foram examinadas na seguinte ordem: (1) semimucosa labial superior; (2) mucosa labial superior; (3) mucosa alveolar superior; (4) gengiva e rebordo superiores; (5) palato duro; (6) palato mole; (7) orofaringe; (8) dorso da língua; (9) bordas laterais da língua; (10) ventre da língua; (11) assoalho bucal; (12) gengiva e rebordo inferiores; (13) mucosa alveolar inferior; (14) mucosa jugal direita e esquerda; (15) mucosa labial inferior; (16) semimucosa labial inferior; e (17) comissuras labiais. As lesões decorrentes de cárie dentária, abscessos periapicais de origem endodôntica e doença periodontal foram excluídas, por não constituírem objeto deste estudo.

A pós terem sido esclarecidos sobre os objetivos do estudo e dos procedimentos a serem realizados, os diretores das instituições assinaram o Termo de Consentimento Livre e Esclarecido, autorizando a partici pação dos internos na pesquisa. Segundo o artigo 92, parágrafo único, do Estatuto da Criança e do Adolescente, o diretor da instituição é equiparado ao guardião, para todos os efeitos de direito. A cada participante foram explicados os objetivos do exame e que poderiam a qualquer momento desistir de fazer parte da pesquisa, sem que isso trouxesse prejuízo em relação ao tratamento estomatológico.

Os examinados que apresentaram lesões de mucosa bucal enecessitaram deexames complementares para diagnóstico conclusivo foram encaminhados à Clínica de Estomatologia da Faculdade de Odontologia da Pontifícia Universidade Católica de M inas Gerais para avaliação e tratamento.

A análise estatística do presente estudo foi baseada nas seguintes etapas: (1) Numeração, tabulação edigitação dos questionários; (2) Análise descritiva (quantidadee percentual) detodas as variáveis do questionário, considerando o grupo dos 231 participantes do estudo; (3) Verificação da associação entre a presença de lesão fundamental e/ou alterações da normalidade ea cor da pele e da prevalência global da pigmentação melânica fisiológica e leucoedema. Baseado na literatura que relata associação entre estas duas alterações da normalidade e a cor da pele, realizamos cálculos para verificar essas potenciais associações. A análise estatística consi stiu demedidas descritivas para as variáveis estudadas. Para isto foram utilizados as frequências e os valores percentuais.

Para a comparação da presença de lesão fundamental e/ou alteração de normalidade versus cor da pele, número médio de lesões fundamentais e número médio dealterações denormalidade versus cor da pele, utilizamos o testenão paramétrico de Kruskall-Walis. A associação entre pigmentação melânica fisiológica e leucoedema versus cor da pele foi feita através do teste Quiquadrado.

Em todos os testes estatísticos utilizados, foi considerado o nível designificância de 5\%. Dessa forma, são consideradas diferenças estatisticamente significantes aquelas cujo valor $p$ foi inferior a 0,05 .

\section{Resultados}

Astabelas 1 e 2 apresentam a distribuição percentual dos examinados em relação à presença de lesões fundamentais ealterações da normalidade.

0 percentual de adolescentes que apresentaram lesões fundamentais foi de 24,24\% (Tabela 1). 
Observa-seque $78,35 \%$ dos examinados apresentaram pelo menos uma alteração da normalidade (Tabela 2).

Dos 231 examinados, 34 (14,7\%) não apre sentaram lesões fundamentais nem alterações de normalidade, $16(6,9 \%)$ apresentaram somente lesões fundamentais, $141(61,0 \%)$ apresentaram somente alterações da normalidadee 40 (17,3\%) apresentaram lesões fundamentais e alterações da normalidade ocorrendo simultaneamente. A Tabela 3 apresenta a relação entre as lesões fundamentais e alterações da normalidade de acordo com a cor da pele do examinado.

Foi observada diferença estatisticamentesignificante na ocorrência de lesões e/ou alterações de normalidade de acordo com a cor da pele do examinado (valor $p<0,05$ ). Pode ser observado que o percentual de adolescentes leucodermas

Tabela 1. Distribuição percentual dos examinados - lesões fundamentais.

\begin{tabular}{lcc}
\hline Lesões fundamentais & Frequência & Percentual \\
\hline Nenhuma & 175 & 75,76 \\
U ma & 48 & 20,78 \\
Duas & 8 & 3,46 \\
\hline
\end{tabular}

Tabela 2. Distribuição percentual dos examinados - alterações de normalidade.

\begin{tabular}{lcc}
\hline $\begin{array}{c}\text { Alterações de } \\
\text { normalidade }\end{array}$ & Frequência & Percentual \\
\hline Nenhuma & 50 & 21,65 \\
Uma & 95 & 41,13 \\
Duas & 63 & 27,27 \\
Três & 20 & 8,65 \\
Quatro & 3 & 1,30 \\
\hline
\end{tabular}

sem lesão fundamental e alteração da normalidade $(28,8 \%)$ é maior que o percentual dos adolescentes melanodermas $(6,5 \%)$ e feodermas $(9,9 \%)$, e que o percentual de adolescentes que apresentam apenas alteração da normalidade é inferior dentre aqueles que são leucodermas (46,6\%) (Tabela 3).

\section{Lesões fundamentais}

Como mostrado na Tabela $1,24,24 \%$ dos examinados apresentaram pelo menos uma lesão fundamental. A Tabela 4 apresenta a distribuição percentual das lesões fundamentais encontradas.

Foram diagnosticadas 64 lesões fundamentais nos 231 examinados, obtendo-se assim uma prevalência total dessas alterações da mucosa bucal de $27,70 \%$, sendo que alguns deles apresentaram mais de uma lesão (Tabela 1). Na população estudada, as lesões fundamentais identificadas foram: placa, erosão, fissura, nódulo, úlcera e pápula.

A placa (32,81\%) (Tabela 4) foi a principal lesão da mucosa encontrada, a maioria de origem traumática, decorrente provavelmente de trauma mecânico provocado por hábito de mastigação crônica da mucosa. Uma delas foi provocada por aparelho ortodôntico e sete pelo uso do tabaco. Três placas removíveis localizadas na orofaringe eram de origem bacteriana.

A erosão (21,88\%) (Tabela 4) foi a segunda alteração mais prevalente. Dos quatorze casos encontrados (Tabela 4), doze foram de origem traumática, provavelmente provocados por hábito de mastigação crônica da mucosa bucal. Uma foi provocada por mordida acidental e outros dois casos localizados na semimucosa labial inferior por variação térmica.

Foram diagnosticados doze casos de fissura $(18,75 \%)$ (Tabela 4), a maioria relacionado com

Tabela 3. Presença de lesão fundamental e/ou alteração de normalidade versus cor da pele.

\begin{tabular}{lrrrr}
\hline \multirow{2}{*}{ Grupo } & \multicolumn{3}{c}{ Cor da pele } & \multicolumn{1}{c}{ Total } \\
\cline { 2 - 4 } & Leucoderma & M elanoderma & \multicolumn{1}{c}{ Feoderma } & \\
\hline Sem lesão e alteração & $21(28,8 \%)$ & $5(6,5 \%)$ & $8(9,9 \%)$ & $34(14,7 \%)$ \\
Apenas lesão & $7(9,6 \%)$ & $3(3,9 \%)$ & $6(7,4 \%)$ & $16(6,9 \%)$ \\
Apenas alteração & $34(46,6 \%)$ & $56(72,7 \%)$ & $51(63,0 \%)$ & $141(61,0 \%)$ \\
Lesão e alteração & $11(15,1 \%)$ & $13(16,9 \%)$ & $16(19,8 \%)$ & $40(17,3 \%)$ \\
Total & $73(100,0 \%)$ & $77(100,0 \%)$ & $81(100,0 \%)$ & $231(100,0 \%)$
\end{tabular}

Valor $p=0,002$. 
a variação térmica (11 casos). Dez casos de nódulo foram identificados (15,63\%) (Tabela 4). Todos eles foram provocados por trauma mecânico, a maioria resultante de brigas entre os internos, sendo um deles provocado pela presença de diastema.

As lesões ulceradas constituíram cinco casos (7,81\%) (Tabela 4); três eram deorigem traumática, provocadas por mordida acidental, e as demais decorreram de infecção herpética recorrente.

As lesões fundamentais menos prevalentes foram as pápulas (3,13\%). Apenas dois casos foram identificados (Tabela 4). Ambos foram submetidos à biópsia excisional e exame anatomopatológico no Laboratório de Estomatologia da Pontifícia Universidade Católica de M inas Gerais. U ma delas foi diagnosticada como hiperplasia focal e a outra como verruga vulgar.

O número médio delesões fundamentais por cor da pele foi maior nos adolescentes feodermas $(0,32)$, seguidos pelos leucodermas $(0,27)$ e melanodermas (0,23); no entanto, apesar de ter sido maior dentre os feodermas, não foi observada diferença estatisticamentesignificanteentre os três grupos (valor $p=0,618$ ).

\section{Alteração da normalidade}

Como pôde ser observado na Tabela 2, $78,35 \%$ dos examinados apresentaram pelo menos uma alteração de normalidade, sendo que $37,23 \%$ apresentaram duas ou mais alterações. Foram identificadas 293 alterações da normalidade. A Tabela 5 apresenta a distribuição percentual dessas alterações.

0 número médio de alterações de normalidade foi maior nos melanodermas $(1,70)$, segui-

Tabela 4. Distribuiç̧ão percentual das lesões fundamentais.

\begin{tabular}{lcc}
\hline Lesões fundamentais & Frequência & Percentual \\
\hline M ácula & 0 & 0,00 \\
Placa & 21 & 32,81 \\
Nódulo & 10 & 15,63 \\
Pápula & 2 & 3,13 \\
Vesícula & 0 & 0,00 \\
Bolha & 0 & 0,00 \\
Erosão & 14 & 21,88 \\
Úlcera & 5 & 7,81 \\
Fissura & 12 & 18,75 \\
Pseudomembranosa & 0 & 0,00 \\
Hiperplásica & 0 & 0,00 \\
\hline
\end{tabular}

dos pelos feodermas $(1,25)$. Os leucodermas foram os que apresentam menor número médio de alterações de normalidade $(0,84)$. Foi observada diferença estatisticamentesignificante quando comparados os três grupos (valor $p=0,000$ ).

\section{Associações}

Foi verificada a potencial associação entre a cor da pele do jovem examinado e a presença de pigmentação melânica fisiológica e leucoedema. As associações foram avaliadas através do teste Qui-quadrado.

O bservou-se associação estatisticamente significanteentrea pigmentação melânica fisiológica ea cor da peledo examinando (valor $p=0,0000$ ). Dos 231 examinados, $131(56,7 \%)$ apresentaram a alteração, sendo 11 deles leucodermas (15,1\% de 73 examinados), 58 feodermas ( $71,6 \% / 81)$ e 62 melanodermas $(80,5 \% / 77)$, portanto, a prevalência de pigmentação melânica fisiológica foi menor nos leucodermas.

Entre a presença de leucoedema e a cor da pele do examinado (valor $p=0,002$ ), também foi observada associação estatisticamente significante. A prevalência da alteração ésuperior nos melanodermas 39,0\% (30/77). Os leucodermasapresentaram-se com 17,8\% (13/73) e os feodermas com $17,3 \%(14 / 81)$.

\section{Discussão}

Os estudos de prevalência fornecem dados sobre a distribuição de uma determinada condição em um grupo populacional específico. Essas informações podem ser utilizadas para o planejamen-

Tabela 5. Distribuição percentual das alterações de normalidade.

\begin{tabular}{lcc}
\hline Alterações de normalidade & Frequência & Percentual \\
\hline Anquiloglossia & 1 & 0,34 \\
Eritema areata migratório & 2 & 0,68 \\
Fossetas da comissura labial & 15 & 5,12 \\
Grânulos de Fordyce & 51 & 17,41 \\
Leucoedema & 57 & 19,45 \\
Língua fissurada & 18 & 6,14 \\
Pigmentação melânica & 131 & 44,71 \\
fisiológica & & \\
Torus mandibular & 5 & 1,71 \\
Torus palatino & 12 & 4,10 \\
Úvula bífida & 1 & 0,34 \\
\hline
\end{tabular}


to de ações coletivas de saúde, bem como para o levantamento de hipóteses a serem testadas em estudos analíticos futuros. Para o diagnóstico correto e tratamento mais adequado, torna-se evidente que o conhecimento das alterações que podem ocorrer na boca, bem como sua etiologia, é de fundamental importância.

N este estudo, optamos por realizar interlocução com vários autores que realizaram pesquisas em populações de faixa etária semelhante que, no entanto, não se encontravam em regime de confinamento. Um fator que limita a comparação de dados é não haver na literatura referenciais teóricos recentes sobre prevalência de alterações nesse grupo específico de adolescentes.

No Brasil, estudos epidemiológicos sobre alterações de mu cosa bucal envolven do adolescentes são raros. Além disso, a falta de padronização na col eta dos dados, diversidade de critérios clínicos de diagnóstico, o uso não uniformizado de termos epidemiológicos, tais como prevalência e incidência, também se constituem em elemento dificultador na comparação dos trabalhos, fato constatado por nós e relatado por Bouquot². Segundo este autor, a prevalência de uma doença é definida como a frequência com que ela ocorre em uma população em um momento particular; a incidência é o número de novos casos de uma determinada doença diagnosticados em um período específico. Assim, todos os estudos, incluindo o presente, que descrevem a frequência deuma alteração através de dados coletados em tempo único fornecem como resultado a prevalência da condição, independentementeda abrangência da amostra populacional.

Uma abordagem sistemática na coleta e no relato dos diagnósticos das alterações da mucosa bucal que possibilite o intercâmbio de informações entre pesquisadores é de fundamental importância.

Quando se considera a prevalência total que inclui lesões fundamentais e alterações da normalidade, o presente estudo encontrou um percentual de 85,2\% (Tabela 3), com 40,68\% (tabelas 1 e 2) dos examinados apresentando ou mais de uma lesão ou mais de uma alteração da normalidade, resultado diferente do encontrado por Bouquot $^{2}$, que em 23.616 pacientes, homens e mulheres, constatou que $10,3 \%$ da população apresentava algum tipo de alteração, com aproximadamente $25 \%$ dos examinados apresentando mais de uma alteração. A diferença observada pode se dever ao fato de que no trabalho desse autor o levantamento foi feito em homens e mulheres de todas as idades e não foram consi- deradas todas as alterações da normalidade, como a pigmentação melânica fisiológica eo leucoedema, que tiveram alta prevalência neste estudo (Tabela 5).

Levantamento epidemiológico, com metodologia mais próxima à utilizada neste trabal ho, foi realizado por Crivelli et al. ${ }^{6}$, que identificaram $33,76 \%$ de crianças e adolescentes apresentando algum tipo de lesão da mucosa bucal. A verificação deuma prevalência superior à encontrada por nós $(24,24 \%$ ) (Tabela 1 ) pode estar associada à inclusão de crianças edelesões periodontaisinflamatórias no trabalho desses autores. Casariego et al. ${ }^{7}$ relataram que da amostra de 301 pacientes obtidas em laboratório de patologia bucal 24,04\% apresentaram lesões. A pesar da não especificação da idade dos pacientes e de diferentes condições da pesquisa, o resultado apresentado foi semeIhante ao encontrado no presente estudo.

$\mathrm{Na}$ literatura, observamos al guns resultados semel hantes como os relatados por Diaz-Gusman eCastellanos ${ }^{8}$, que avaliaram, entrejaneiro de 1982 e julho de 1986, 7.297 pacientes com 15 anos ou mais, encontrando prevalência aproximada de $13 \%$ dos examinados apresentando lesões de mucosa bucal; e por Nair et al. ${ }^{9}$, que ao examinarem 550 pessoas de 2 a 60 anos de idade identificaram $14 \%$. No entanto, muitos relatos apresentam discrepância, provavelmente devido à falta de padronização com relação à metodologia, como a inclusão de doenças periodontais inflamatórias e abscessos periapicais, exclusão de algumas alterações da normalidade como leucoedema e pigmentação melânica fisiológica, faixas etárias queincluem crianças, adolescentes eadultos ao mesmo tempo, critérios diagnósticos diversos e ainda diferentes áreas geográficas.

As alterações da normalidade neste estudo tiveram prevalência de 78,35\% (Tabela 2). Bryn ${ }^{10}$, examinando estudantes de 7 a 13 anos de idade, constatou 33,54\%. A discrepância pode ser explicada pela diferença das faixas etárias estudadas, pelo fato de algumas alterações aumentarem a sua prevalência com a idade e pela diferença de metodologia, com a inclusão no presente trabaIho de todas as alterações da normalidade.

A lesão mais prevalentenesteestudo foi a placa $(32,81 \%)$ (Tabela 5). Os adolescentes que as apresentaram, as quais foram provocadas pelo uso do fumo, tiveram esclarecimento a respeito dos efeitos do tabaco no aparecimento dessas lesões e orientados a abandonar o hábito.

Um estudo sobre prevalência de lesões traumáticas da mucosa bucal em 3.779 adolescentes e adultos jovens institucionalizados foi realizado 
por Van Wyk et al. ${ }^{11}$. A maioria deles encontravasena faixa etária de 16 e 17 anos. A prevalência de masti gação crônica da mucosa bucal foi de 4,6\%. Os autores examinaram comparativamente 555 alunos da mesma idadeque não viviam em instituições; nenhuma lesão por mastigação foi encontrada. Concluíram que a maior prevalência de lesões em alunos institucionalizados se deveu ao estresse e às condições psicológicas e emocionais desse grupo. O bservamos quea maioria das placas e erosões identificadas nesta pesquisa decorreu desse hábito. A população participante deste estudo encontra-se sob estresse constante, provocado por brigas, rebeliões e fugas.

Alguns casos de erosão (2/14) e quase todos os casos de fissura (11/12) estavam relacionados à variação térmica. Os que as apresentaram foram medicados por nós com pomada de Dexametasona e Dexpantenol, e para prevenirem a ocorrência das lesões foram orientados a usar algum tipo de proteção.

Deve-se ressaltar que estudos transversais, isto é, realizados em tempo único, podem subestimar a real prevalência delesões recorrentes como herpes e ulceração aftosa. Kleinman et al. ${ }^{3}$ fizeram levantamento epidemiológico em 39.206 escolares, com idade entre 5 e 17 anos. Foi perguntado a todas as crianças e adolescentes se houve história de ocorrência de lesões desencadeadas pelo frio, presença de vesículas precedidas por febre, eainda presença de lesõesulcerativas; 33\% deles relataram história pregressa de herpes labial recorrentee $37 \%$ deulceração aftosa recorrente. Q uando foi considerada a identificação clínica do herpes labial recorrente, os autores relataram $0,78 \%$ dos examinados apresentando a lesão, resultado semelhanteao desteestudo, 0,86\% (lesão ulcerada - Tabela 4), e diferente dos resultados encontrados por Cerri e Genovese $e^{12}$ que, ao examinarem 326 reeducandos do Sistema Penitenciário do São Paulo, encontraram a condição em 1,8\% dos examinados. Embora os estudos transversais sobre a prevalência de ulcerações aftosas recorrentes variem entre 1,5\% e 7,0\% nas diversas populações, em nossos estudos não foi constatado nenhum caso, provavelmente devido a fatores determinantes da lesão.

A prevalência de verruga vulgar provocada pelo papilomavírus humano (H PV) em nossos estudos foi de $0,43 \%$ (1/231) e ocorreu em um adolescente de 17 anos. Summersgill et al. ${ }^{13}$ realizaram uma pesquisa com crianças e adolescentes quanto à frequência do H PV. A taxa de soropositividade para o vírus em adolescentes (acima de 13 anos) foi de 5,2\%. A população estuda- da não apresentou evidência de infecção oral pelo HPV. Os autores concluíram que essa infecção poderia ocorrer na infância, desaparecer e posteriormente haver uma reinfecção, ou ainda a hipótese de haver uma infecção persistente com carga viral quenão poderia ser detectada nos testes laboratoriais.

As duas alterações da normalidade mais prevalentes foram a pigmentação melânica fisiológica $(44,71 \%$ ) (Tabela 5) com $56,7 \%$ dos adolescentesacometidos, sen do esta mais preval entenos melanodermas ( $80,5 \%)$, e o leucoedema ( $19,45 \%$ ) (Tabela 5), com 24,7\% dos examinados apresentando a alteração, sendo esta mais comum também nos melanodermas $(39,0 \%)$. Eleutério ${ }^{14}$ realizou um estudo em 991 crianças e adolescentes entre 5 e 14 anos de idade para a verificação de pigmentação melânica fisiológica. A frequência encontrada na raça branca foi de $11,8 \%$ a $13,1 \%$; na raça amarela foi de $64 \%$ a 75,9\%; enos deraça negra, de $91,3 \%$ a $95 \%$. 0 autor ressaltou que a pigmentação melânica fisiológica aumenta quantitativamente com a idade. No entanto, a prevalência das alterações em negros relatada por ele foi maior, quando comparada aos nossos relatos (80,5\%), em que examinamos uma população com idade superior. Em relação aos adolescentes da raça branca, o resultado foi semelhante(15,1\%) (Tabela 5). N este estudo, foram observadas diferenças estatisticamente significantes quando essa alteração foi comparada à cor da pele $(p=0,000)$. De acordo com Axéll e Henricsson ${ }^{15}$, o leucoedema corre mais comumente em negros adultos, com aproximadamente $90 \%$ de prevalência. $\mathrm{Na}$ presente pesquisa, quando a presença do leucoedema foi comparada com a cor da pele dos examinados, observou-se asso ciação estatisticamente significante $(p=0,002)$. A alteração foi mais prevalentenos melanodermas, com 39,0\%, não existindo diferença significante entre leucodermas e fenodermas, com $17,8 \%$ e $17,3 \%$, respectivamente. A discrepância nos resultados pode estar relacionada a critérios clínicos de diagnóstico, diversidade de grupos populacionais pesquisados efatores determinantes.

A prevalência de língua fissurada neste estudo foi de $6,14 \%$ e de eritema areta migratório, 0,68\% (Tabela 5). A literatura mostra-se divergente quanto à prevalência dessas alterações. Sawyer et al. ${ }^{16}$, em 2.203 escolares nigerianos de 10 a 19 anos deidade, relataram prevalência delíngua fissurada em $0,8 \%$ e de eritema areta migratório em $0,2 \%$ dos examinados. Sedano et al. ${ }^{17}$ realizaram um estudo com crianças e adolescentes mexicanos de 5 a 14 anos e meio de idade e relata- 
ram $15,7 \%$ de língua fissurada e $0,98 \%$ de eritema areta migratório. Crivelli et al. ${ }^{6}$ investigaram 660 crianças eadolescentes de 3 a 13 anos de idade em relação às anomalias da língua. A prevalência de língua fissurada foi de $0,45 \%$ ea deeritema areata migratório, 3,63\%. Kovac-Kovacic e Skaleri $c^{18}$ encontraram presença de língua fissurada em $21,1 \%$ da população estudada.

$\mathrm{Na}$ literatura, a prevalência de língua fissurada relatada variou entre $0,45 \%$ e $21,1 \%$ edeeritema areata migratório entre $0,2 \%$ e 5,67\%, sendo tema de pesquisas de vários autores ${ }^{19-33}$. A divergência na prevalência dessas alterações da normalidade pode se explicar pelo fato de as populações estudadas serem distintas quanto às faixas etárias e a fatores que determinam as alterações.

A associação entre língua fissurada e eritema areata migratório está bem documentada por vários autores 1,17,24,34,35. Rahamimoff e M uhsam $^{24}$, após um período de acompanhamento de oito anos, relataram transformação de eritema areata migratório em língua fissurada em 41 pessoas examinadas e a ocorrência simultânea das duas alterações em oito delas. Este aspecto foi considerado pelos autores como uma indicação de que o eritema areata migratório poderia se transformar em língua fissurada. Em nossos estudos, encontramos apenas um adolescente com a ocorrência simultânea das duas condições.

Anquiloglossia teveprevalência de $0,34 \%$ neste estudo, resultado diferente dos encontrados por Sedano ${ }^{36}$, que obteve prevalência de $0,1 \%$ em crianças e adolescentes de 6 a 15 anos de idade, por Sedano et al. ${ }^{17}$, que relataram prevalência de 0,83\% em uma população entre 5 e 14 anos e meio de idade, e por Crivelli et al. ${ }^{37}$, que relataram prevalência de 1,98\% em crianças e adolescentes de 3 a 13 anos de idade e semelhante às encontradas por M umcu et al..$^{38}(0,3 \%)$, que pesquisaram lesões de mucosa bucal na população turca. Essa alteração parece não estar associada à faixa etária; no entanto, para uma conclusão, mais estudos são necessários.

Kovac-Kovacic e Skaleric ${ }^{18}$ encontraram prevalência de grânulos de Fordyce de $49,7 \%$ na população pesquisada, composta principalmente por adultos, percentual maior do que o relatado neste estudo, 17,41\% (Tabela 5). Na literatura, não encontramos trabal hos sobre a prevalência dessa condição exclusivamente em adolescentes, portanto, relatos deprevalência mais alta em adultos podem indicar uma relação com a idade.

Em nossa pesquisa, verificamos prevalência de fosseta da comissura labial de 5,12\% (Tabela 5), resultado semelhante ao apresentados por
Sedano et al. ${ }^{17}$, que realizaram um estudo com crianças e adolescentes de 5 a 14 anos e meio de idade, relatando prevalência de 5,28\%. Estes autores observaram ainda que a alteração tende a aumentar com a idade.

Sawyer et al. ${ }^{16}$ estudaram a prevalência de alterações da mucosa bucal em 2.203 escolares de 10 a 19 anos de idade e encontraram $4,5 \%$ de torus palatino e $1,9 \%$ detorus mandibular. A prevalência relatada pelos autores foi semelhante à encontrada no presente estudo: torus palatino, 4,10\%; e torus mandibular, $1,71 \%$ (Tabela 5 ).

Osresultados apresentados nestetrabal ho nos levam a refletir sobrea importância de uma semiotécnica bem conduzida, alicerçada em conhecimentos amplos sobre as al terações e doen ças que se manifestam na mucosa bucal. 0 comprometimento do cirurgião-dentista com a determinação da prevalência das alterações bucais em adolescentes, particularmente naqueles que vivem confinados, o leva como profissional da área de saúdea planejar atendimento, individual ou coletivo, dessas populações. Os resultados poderão ainda servir de base para futuros estudos sobre a prevalência de alterações da mucosa bucal e de seus determinantes em populações semelhantes.

\section{Conclusões}

Osresultados do presenteestudo nos trazem al gumas informações sobre prevalência de lesões fundamentais e alterações da normalidade em adolescentes confinadosenospermitem concluir que elas são diferentes das encontradas em populações de mesma faixa etária e em outro ambiente.

0 estudo realizado foi o de corte transversal, isto é, em tempo único; assim, a verdadeira prevalência de lesões recorrentes como herpes labial e ulcerações aftosas pode ter sido subestimada.

Algumas lesões como placa, erosão e nódulo podem ter sido decorridas das condições psicológicas e emocionais desse grupo e do uso de tabaco. Esta éa única droga permitida nos centros deinternação.

0 percentual de adolescentes que apresentaram lesão fundamental e alteração da normalidade foi de 85,3\% (Tabela 3), a presença de lesão fundamental foi de $24,24 \%$ (Tabela 1) eas dealteração da normalidade foi de 78,35\% (Tabela 2).

Os adolescentes que não apresentaram lesão fundamental nem alteração da normalidade e apenas lesão fundamental foram os leucodermas, com 28,8\% e 9,6\%, respectivamente (Tabela 3). Os que apresentaram maior prevalência de 
alterações da normalidade foram os melanodermas, com 72,7\% (Tabela 3), e maior prevalência de lesões fundamentais e alterações da normalidade ocorrendo simultaneamente foram os feodermas, com 19,8\% (Tabela 3). Este resultado nos permite observar diferença estatisticamente significante quando comparamos a presença de lesões e/ou alterações de normalidade e a cor da pele do examinado (valor $p<0,05$ ).

Em relação ao número médio de lesões fundamentais por cor da pele, foi maior nos adolescentes, não foi observada diferença estatisticamen- tesignificanteentreos três grupos (valor $p=0,618$ ). Quanto às alterações de normalidade, houve diferença estatisticamentesignificantequando comparados os três grupos (valor $p=0,000$ ).

Os resultados apresentados foram diferentes dos encontrados por outros autores, portanto, mais estudos são necessários para a verificação da prevalência das alterações de mucosa bucal e de seus fatores determinantes na população em geral ede modo particular em adolescentes, com a particularidade de viverem confinados.

\section{Colaboradores}

RA Hipólito trabalhou na concepção, no deline amento, análise, redação e interpretação dos dados. CR M artins trabalhou na concepção, no delineamento e na redação final.

\section{Referências}

1. Pereira MG. Epidemiologia: teoria e prática. Rio de Janeiro: Guanabara-Koogan; 1995.

2. Bouquot J. Common oral lesions found during a mass screening examination. Am Dent Assoc 1986; 112(1):50-57.

3. Kleinman DV, Swango PA, Pindborg JJ. Epidemiology of oral mucosal lesions in United States schoolchildren: 1986-87. Community Dent Oral Epidemiol 1994; 22(4):243-253.

4. SB 2000 - Ação Complementar - Prevalência de fatores de risco - Lesões cancerizáveis e câncer da boca - M inas Gerais, 2000. [acessado 2007 mar 12]. Disponível em: http://www.saude.gov.br/programas/ bucal/sb2000.htm

5. Neville BW, Damm DD, Allen CM, Bouquot JE. Oral \& Maxillofacial Pathology. Philadelphia: W. B. Saunders Company; 1995.

6. Crivelli MR, Muhlmann M, Adler I, Cornicelli JC. Prevalencia de patologia bucal en niños. Rev Assoc Odont 1986; 74(3):81-82.

7. Casariego Z, Drut R, Micinquevich S. Frecuencia y tipos de lesiones en um laboratório de patologia oral. Odontol Bonaer 1995; 17(52):6-7.

8. Dias-Guzman L, Castellanos JL. Lesions of the oral mucosa: epidemiological study of 1297 patients. Rev ADM 1991; 48(2):75-80.

9. Nair RG, Samaranayake LP, Philipsen RGB. Prevalence of oral lesions in a selected Vietnamese population. Int Dent J 1996; 46(1):48-51.

10. Bryn AL. Estudo das alterações e lesões dos tecidos moles da boca em crianças de 7 a 13 anos na cidade de São Paulo, 1992 [dissertação]. São Paulo: Faculdade de Odontologia, Universidade de São Paulo; 1992. 
11. Van Wyk CW, Staz J, Farman AG. The chewing lesion of the cheeks and lips: its features and prevalence among a selected group of adolescents. J Dent 1977; 5(3):193-199.

12. Cerri A, Genovese WJ. Alterações da mucosa bucal: estudo epidemiológico da sua prevalência em reeducandos do sistema penitenciário do eEstado de São Paulo. RGO 1991; 39(3):218-220.

13. Summersgill KF, Smith EM, Levy BT, Allen JM, Haugen TH, Turer LP. Human papillomavirus in the oral cavities of children and adolescents. Oral Surg Oral Med Oral Pathol Oral Radiol Endod 2001; 91(1):62-69.

14. Eleutério D. Contribuição para o estudo clínico da pigmentação melânica fisiológica intraoral em crianças selecionadas em três grupos étnicos distintos: branco, amarelo e negro. Rev Fac Farm Odont. Araraquara 1969; 3(1):19-47.

15. Axéll T, Henricsson V. Leukoedema: an epidemiologic study with special reference to the influence of tobacco habits. Community Dent Oral Epidemiol 1981; 9:142-146.

16. Sawyer RD, Taiwo EO, Mosadomi A. Oral anomalies in Nigerian children. Community Dent Oral Epidemiol 1984; 12(6):269-273.

17. Sedano HO, Carreon Freyre I, Garza de la Garza ML, Gomar Franco CM, Grimaldo Hernandez C, Hernandes Montoya ME et al. Clinical orodental abnormalities in M exican children. Oral Surg Oral Med Oral Pathol 1989; 68(3):300-311.

18. Kovac-Kovacic M, Skaleric $V$. The prevalence of oral mucosal, lesions in a population in Ljublyana Slovenia. J Oral Pathol Med 2000; 29(7):331-335.

19. Halperin V, Kollas $S$, Jefferis $K R$, Huddleston SO, Robinson HBG. The occurrence of Fordyce spots, benign migratory glossitis, median rhomboid golssitis and fissured tongue in 2478 dental patients. J Oral Surg 1953; 6(6):1072-1077.

20. Hume WJ. Geographic stomatitis: a critical review. J. Dent 1975; 3(1): 25-43.

21. Marks R, Czarny D. Geographic tongue: sensitivity to the environment. J Oral Surg 1984; 58(2):156-159.

22. Brooks JK, Balciunas BA. Geographic stomatitis: review of the literature and report of five cases. J Am Dent Assoc 1987; 115(3):412-414.

23. Sigal MJ, Mock D. Syntomatic benign migratory glossitis: report of two cases and literature review. Pediatr Dent 1992; 14(6):392-396.

24. Rahamimoff $P$, Muhsam HV. Some observations on 1,246 cases of geographic tongue. Am J Dis Child 1957; (93):519-525.

25. Kullaa-M ikkonen A. Studies on lingua fissurata. Proc Finn Dent Soc 1986; 82(Supl.4):1-48.

26. M eskin LH, Redman RS, Gorlin RJ. Incidence of geographic tongue among 3668 students at the University of Minnesota. J Dent Res 1963; 42(3):895.
27. Richardson ER. Incidence of geographic tongue and median rhomboid glossitis in $3319 \mathrm{Negro} \mathrm{col-}$ lege students. J Oral Surg 1968; 26(5):623-625.

28. Gallina L. Indagine clinico-statistica sulla frequenza della lingua scrotalle, della lingua geografica, della lingua near villosa, della glossite rombica mediana, dell'anquiloglossia e del torus palatinus in 3274 stomatopazienti. Rass Int Stomat Prat 1968; 19(4):261-267.

29. Kullaa-Mikkonen A, Mikkonen M, Kotilainen R. Prevalence of different morphologic forms of human tongue in young Finns. Oral Surg Oral Med Oral Pathol 1982; 53(2):152-156.

30. Bánóczy J, Rigó 0 , Albrecht M. Prevalence study of tongue lesions in a Hungarian population. Community Dent Oral Epidemiol 1993; 21(4):224-226.

31. Redman RS. The prevalence of geographic tongue, fissured tongue, median rhomboid glossitis, and hairy tongue among 3,611 M innesota school children. J Oral Surg 1970; 30(3):390-395.

32. Ghose LJ, Baghdady VS. Prevalence of geographic and plicated tongue in 6090 Iraque schoolchildren. Community Dent Oral Epidemiol 1982; 10(4):214-216.

33. Kullaa-Mikkonen A. Familial study of fissured tongue. Scand J Dent 1988; 96(4):366-375.

34. Chosack A, Zadik D, Eidelman E. Prevalence of scrotal tongue and geographic tongue in 70359 Israeli schoolchildren. Community Dent Oral Epidemiol 1974; 5:253-257.

35. Aboyans V, Ghaemmaghami $A$. the incidence of fissured tongue among 4,009 Irarian dental outpacients. J Oral Surg 1973; 36(1):34-38.

36. Sedano HO. Congenital oral anomalies in Argentinian children. Community Dent Oral Epidemiol 1975; 3(2):61-63.

37. Crivelli MR, Aguas S, Quarracino C, Adler I, Brausnstein S. Prevalência de anomalias linguales em niños. Rev Assoc O dont 1990; 78(2):74-77.

38. Mumcu G, Cimilli H, Sur H, Hayran O, Atalay T. Prevalence and distribution of oral lesions: a crosssectional study in Turkey. Oral Dis 2005; (21):81-87.

Artigo apresentado em 14/11/2007

Aprovado em 19/05/2008

Versão final apresentada em 06/08/2008 